Extent and Magnitude of Activations in Hippocam pal ROI Across T1, T2 and $\mathrm{T} 3 \mathrm{fMRIS}$

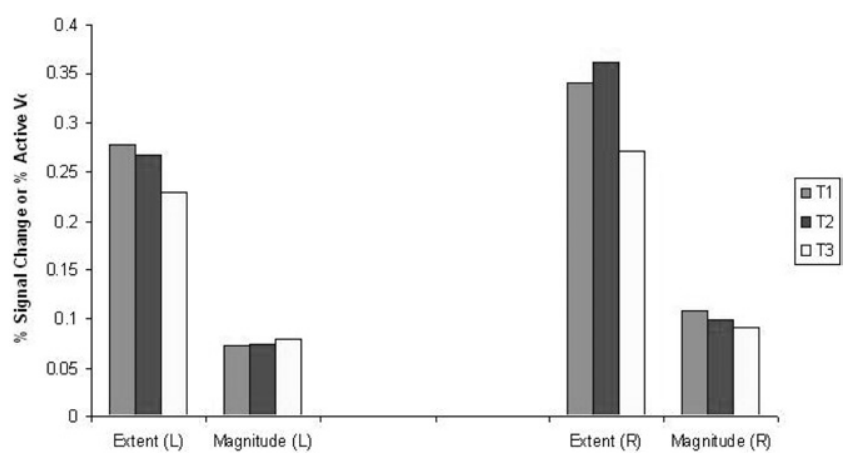

Atri et al. Feasibility and Test-retest Reliability of fMRI in an Alzheimer's Disease Clinical Trial

\section{IC-P2-074 DIFFERENTIATING AMNESTIC MCI CONVERTING TO PROBABLE AD FROM STABLE AMNESTIC MCI USING FDG-PET AND AN AD- RELATED HYPOMETABOLISM OVERLAP INDEX}

Napatkamon Ayutyanont ${ }^{1,2}$, Kewei Chen ${ }^{1,2}$, Xiaofen Liu ${ }^{1,2}$, Cole Reschke ${ }^{1,2}$, Wendy Lee ${ }^{1,2}$, Dan Bandy ${ }^{1,2}$, Gene E. Alexander ${ }^{3,2}$, William J. Jagust ${ }^{4}$, Robert A. Koeppe ${ }^{5}$, Norman L. Foster ${ }^{6}$, Eric M. Reiman ${ }^{7,3},{ }^{1}$ Banner Alzheimer's Institute, Phoenix, AZ, USA; ${ }^{2}$ Arizona Alzheimer's Consortium, Phoenix, AZ, USA; ${ }^{3}$ University of Arizona, Tucson, AZ, USA; ${ }^{4}$ University of California, Berkeley, CA, USA; ${ }^{5}$ University of Michigan, Ann Arbor, MI, USA; ${ }^{6}$ University of Utah, Salt Lake City, UT, USA; ${ }^{7}$ Banner Alzheimer's Institute, Arizona Alzheimer's Consortium, Translational Genomics Research Institute, Phoenix, AZ, USA. Contact e-mail: Napatkamon.Ayutyanont@bannerhealth.com

Background: Alzheimer's disease (AD) is characterized by a characteristic pattern of reductions in the cerebral metabolic rate for glucose (CMRgl). We recently introduced use of an AD-Related Hypometabolism Overlap Index to determine the percentage of previously characterized AD-related hypometabolic brain voxels that are hypometabolic in an individual subject's fluorodeoxyglucose positron emission tomography (FDG-PET) image and help distinguish between probable AD patients, amnestic mild cognitive impairment (MCI) patients and normal controls (NC). The objective of this study is to evaluate the ability of our proposed Overlap Index to distinguish between probable AD patients, MCI patients who convert to probable $\mathrm{AD}$ within the next 18 months (MCIc), MCI patients who have so far remained stable (MCIs) and $\mathrm{NC}$ from the AD Neuroimaging Initiative (ADNI). Methods: The Overlap Index was computed in $74 \mathrm{AD}$ patients, 14 MCIc, 124 MCIs patients, and $82 \mathrm{NC}$ as follows: First, statistical parametric mapping (SPM5) was used to generate individual cerebral hypometabolism z-score maps, comparing each person to a database of 82 NC. Next, the individual hypometabolism map was superimposed on an AD-related hypometabolic map generated from a previous study (Alexander et al, 2002). Finally, the Overlap Index was computed as $\mathrm{V}_{\mathrm{ADI}} \mathrm{I}$ $\mathrm{V}_{\mathrm{AD}} \times 100 \%$, such that $\mathrm{V}_{\mathrm{ADI}}$ is the hypometabolic volume in both previous $\mathrm{AD}-\mathrm{NC}$ group comparison and the subsequent individual-NC comparison, and $\mathrm{V}_{\mathrm{AD}}$ is the hypometabolic volume in only the previous $\mathrm{AD}-\mathrm{NC}$ group comparison. Results: The Overlap Index significantly distinguished the four subject groups (ANOVA $\mathrm{P}=8.8 \mathrm{e}-15$ ). As predicted, the overlap index had the following rank order: $\mathrm{AD}>\mathrm{MCIc}>\mathrm{MCIs}>\mathrm{NC}$ (ANOVA with linear trend $\mathrm{P}=3.9 \mathrm{e}-16$ ). Moreover, the Overlap Index significantly distinguished between the MCIc group and the MCIs group ( $\mathrm{t}$-test $\mathrm{P}=0.013$ ). Conclusions: Our preliminary findings suggest that the AD Overlap Index can help identify hypometabolic patterns consistent with $\mathrm{AD}$ and help differentiate MCI patients converting to $\mathrm{AD}$ from stable MCI patients without the need to correct for multiple comparisons.

\section{IC-P2-075 USE OF REGIONAL THICKNESS MEASURES TO PREDICT DECLINE IN QUESTIONABLE AD DEMENTIA: COMPARISON TO STANDARD VOLUMETRIC MEASURES}

Akram Bakkour ${ }^{1}$, Bruce Fischl ${ }^{1}$, John C. Morris ${ }^{2}$, Randy L. Buckner ${ }^{3}$, Bradford C. Dickerson ${ }^{1},{ }^{1}$ Massachusetts General Hospital, Charlestown, MA, USA $;{ }^{2}$ Washington University, St. Louis, MO, USA; ${ }^{3}$ Harvard University, Cambridge, MA, USA. Contact e-mail: akram@nmr.mgh.harvard.edu

Background: We previously used exploratory analyses to determine that clinically mild Alzheimer's disease (AD) is reliably associated with a "cortical signature" of thinning in a specific set of regions in medial (MTL), inferior (ITG), and polar temporal (TP), inferior, superior (SPL), and medial parietal, and inferior and superior frontal cortices. We sought to determine whether the pattern of $\mathrm{AD}$-related thinning is present in individuals with questionable dementia of the Alzheimer type (QAD) prior to mild $\mathrm{AD}$ and whether a greater degree of regional thinning predicts mild AD dementia. Methods: Participants included 49 older adults with QAD (Clinical Dementia Rating $(C D R)=0.5)$ at the time of structural MRI scanning. Nine regions of interest (ROIs) identified from an exploratory analysis comparing a separate sample of Older Controls to mild $\mathrm{AD}(\mathrm{CDR}=1)$ patients were used to measure $\mathrm{AD}$ signature cortical thickness in these participants. We also measured "mean AD signature" thickness across all 9 regions. In addition, mean thickness across the entire cortex, and whole brain, hippocampal and entorhinal volumes were calculated. Longitudinal clinical follow-up after scanning was used to classify participants as progressors to $\mathrm{CDR}=1$ or non-progressors. Results: Longitudinal follow-up revealed that 20 participants converted to mild $\mathrm{AD}$ dementia after approximately 2.5 years. At baseline, these QADs showed milder thinning in the cortical ROIs as well as milder hippocampal and entorhinal atrophy than is typically seen in mild AD. Compared to the non-progressors, the progressors showed thinning in MTL, ITG, TP and SPL. Effect sizes for group differences were higher for many regional thickness as compared to volume measures. Using receiver operating characteristic curves, mean $\mathrm{AD}$ signature cortical thickness was the best at predicting progression to mild AD with $83 \%$ sensitivity and $65 \%$ specificity as compared to entorhinal volume, which achieved $72 \%$ sensitivity and $65 \%$ specificity. Conclusions: Thinning in specific cortical areas known to be affected by $\mathrm{AD}$ is detectable in individuals with QAD and predicts conversion to mild $\mathrm{AD}$ dementia. This method could be useful for identifying individuals at relatively high risk for progression from QAD to mild AD dementia within a few years, which may be of use in clinical trials.

\section{IC-P2-076 WHITE MATTER VOLUME IN INDIVIDUALS AT INCREASED RISK FOR ALZHEIMER'S DISEASE}

C.E. Beauregard, Catherine Cristinzio, Guillermo Verduzco, Susan Spear Bassett, Johns Hopkins School of Medicine, Baltimore, MD, USA. Contacte-mail: ccristinzio@hotmail.com

Background: While much of structural imaging has focused on gray matter, the quantification of white matter integrity is of interest not only because of the noted white matter pathology seen in Alzheimer's disease (AD), but also because of the apparent breakdown of myelin in AD. To date there has been no longitudinal study of white matter integrity within asymptomatic samples at elevated risk for late-onset AD, the purpose of this study. Methods: Cognitively normal participants age 50 years and above were chosen from an ongoing longitudinal study, comparing those with increased genetic risk for $\mathrm{AD}$ to matched controls. 95 of the high-risk and 91 of the controls had structural scans at baseline (Time 1) and 87 high-risk and 74 controls at three-year follow-up (Time 2). Participants were imaged at each time point on the same 1.5 Tesla Philips Intera-NT system equipped with Galaxy gradients $(66 \mathrm{mT} / \mathrm{m}$ at $110 \mathrm{mT} / \mathrm{m} / \mathrm{s})$. High resolution anatomical images of the brain for structural measurements were acquired using a $\mathrm{T}_{1}$-weighted, 3D MP-RAGE (Magnetization Prepared Rapid Acquisition Gradient Echo) sequence with the following parameters: $\mathrm{TR}=8.6 \mathrm{~ms}, \mathrm{TE}=3.9 \mathrm{~ms}, \mathrm{FOV}=240 \mathrm{~mm}, \theta=8^{\circ}$, matrix size $=256 \times 256$, slice thickness $=1.5 \mathrm{~mm}, 124$ slices). Pre-processing was performed using a semi-automated method, followed by the manual removal 